20

\title{
Численное моделирование миграции фотонов в однородных и неоднородных цилиндрических фантомах
}

\author{
(C) А.Ю. Потлов, С.В. Фролов, С.Г. Проскурин \\ Биомедицинская техника, Тамбовский государственный технический университет, \\ 392000 Тамбов, Россия \\ e-mail: zerner@yandex.ru
}

Поступила в редакцию 28.12.2019 г.

В окончательной редакции 31.01.2020 г.

Принята к публикации 28.022020 г.

\begin{abstract}
Описаны особенности распространения низкокогерентного импульсного излучения в фантомах мягких биологических тканей (кровенасыщенные ткани мозга, молочной железы ит.п.). Проведено сравнение результатов математического моделирования миграции фотонов на основе диффузионного приближения к уравнению переноса излучения и метода статистических испытаний Монте-Карло. Подтверждено, что нормированный максимум фотонной плотности (НМФП) (photon density normalized maximum, PDNM) в относительно однородных сильно рассеивающих средах движется в направлении центра исследуемого объекта. При наличии неоднородностей характер движения НМФП меняется, поглощающая неоднородность в сильно рассеивающей среде смещает траекторию движения НМФП в направлении точки, симметричной центру этой неоднородности относительно центра исследуемого объекта. В случаях с рассеивающей неоднородностью НМФП движется в направлении ее центра.
\end{abstract}

Ключевые слова: сильно рассеивающие среды, миграция фотонов, уравнение переноса излучения, диффузионное приближение, метод Монте-Карло, медицинские фантомы, нормированный максимум фотонной плотности.

DOI: $10.21883 /$ OS.2020.06.49417.33-20

\section{Введение}

Фотоны в процессе диффузионного распространения через биологические ткани испытывают многочисленные акты взаимодействия (поглощение, рассеяние, преломление) на всей протяженности траекторий своего движение, начиная от точки инжектирования и заканчивая точкой детектирования [1-4]. В связи с этим диффузионно прошедшее через исследуемый объект оптическое излучение несет полезную информацию о множестве элементарных участков в составе этого объекта [2,5-7]. Однако для того чтобы восстановить оптические свойства каждого такого участка и сформировать на их основе теоретические пространственные распределения коэффициентов поглощения, $\mu_{a}(x, y, z)$, и рассеяния, $\mu_{s}(x, y, z)$, для всех точек исследуемого объекта с координатами $x, y, z$, необходимо решить обратную задачу $[1,6]$. С теоретической точки зрения процесс решения обратных задач для методов оптической визуализации, в частности различных модификаций метода диффузионной оптической томографии, хорошо известен и детально проработан [1,8-10]. Однако с практической точки зрения нелинейности траекторий фотонов, влияние одних оптических характеристик на другие (например, параметра анизотропии, $g(x, y, z)$, на коэффициент рассеяния, $\left.\mu_{s}(x, y, z)\right)$, сложности с учетом геометрии границ исследуемого объекта, высокий уровень шумов и т. п. делают процесс решения обратной задачи ресурсоемким [7,11], а получаемые пространственные распределения - размытыми [3,12-15].
Актуальным является выявление особенностей распространения низкокогерентного излучения через мягкие биологические ткани и использование этих особенностей для упрощения и уточнения процесса решения обратной задачи $[9,10,13]$. При этом выявление особенностей удобнее всего производить на основе исследования оптически мутных тканеимитирующих фантомов, сходных с реальными биологическими объектами по средним значениям оптических характеристик, но обладающих более простым и заранее известным строением [12-15].

Целью данной работы является анализ влияния оптических свойств и геометрического расположения крупных поглощающих и рассеивающих неоднородностей в исследуемом тканеимитирующем фантоме на формирование времяразрешенного сигнала (ВФРТ - временные функции рассеяния точки, TPSF - time point spread function) на границах исследуемого объекта.

\section{Моделирование миграции фотонов в сильно рассеивающих средах на основе диффузионного приближения к уравнению переноса излучения}

Диффузионное приближение к уравнению переноса излучения (УПИ) довольно точно описывает баланс энергии в сильно рассеивающих средах, таких как био- 
логические ткани или их фантомы $[3,8,16]$. Плотность потока фотонов для множества моделируемых точек с координатами $x, y, z$ может быть представлена как функция времени или как функция частоты [17]. Цели данной работы наиболее соответствует диффузионное приближение к УПИ для ультракоротких импульсов (функция времени) следующего вида [10]:

$$
\begin{aligned}
& \frac{v_{\text {object }}}{c_{0}} \frac{\partial \phi(x, y, z, t)}{\partial t}-D(x, y, z) \nabla^{2} \phi(x, y, z, t) \\
& +\mu_{a}(x, y, z) \phi(x, y, z, t)=I(x, y, z, t), \quad \forall x, y, z \in \Omega,
\end{aligned}
$$

где $c_{0}-$ скорость света в вакууме; $v_{\text {object }}-$ средневзвешенная величина относительного показателя преломления для толщи исследуемой биологической ткани $(\Omega)$ и ее границы $(\partial \Omega) ; \quad D(x, y, z)$ - коэффициент диффузии для множества точек с координатами $x, y, z ; \phi(x, y, z, t)$ - плотность фотонов в точках с координатами $x, y, z$ в момент времени $t$; $I(x, y, z, t)=\delta\left(x_{0}, y_{0}, z_{0}, t_{0}\right)$ - дельта-функция источника фотонов [18-20]. Коэффициент диффузии $[3,10]$ вычисляется на основе коэффициента поглощения, $\mu_{a}(x, y, z)$, и редуцированного коэффициента рассеяния, $\mu_{s}^{\prime}(x, y, z)$ :

$$
\begin{aligned}
D(x, y, z) & =\frac{1}{3\left[\mu_{a}(x, y, z)+(1-g(x, y, z)) \mu_{s}(x, y, z)\right]} \\
& =\frac{1}{3\left[\mu_{a}(x, y, z)+\mu_{s}^{\prime}(x, y, z)\right]} .
\end{aligned}
$$

Описание потока фотонов на границе моделируемой биологической ткани осуществляется с использованием граничного условия III-го рода $[10,21]$ :

$$
\begin{aligned}
& \phi(x, y, z, t)+2 D(x, y, z) \frac{\frac{2}{1-R_{0}}-1+\left|\cos \left(Q_{c}\right)\right|^{3}}{1-\left|\cos \left(Q_{c}\right)\right|^{2}} \\
& \times \frac{\partial \phi(x, y, z, t)}{\partial \mathbf{n}(x, y, z)}=0, \quad \forall x, y, z \in \partial \Omega, \quad x, y, z \notin q,
\end{aligned}
$$

где $\mathbf{n}(x, y, z)$ - внешняя нормаль к границе исследуемого объекта $\partial \Omega$ для всех точек с координатами , кроме точки источника фотонов $q=x_{0}, y_{0}, z_{0} ; R_{0}$ и $Q_{c}-$ коэффициенты, характеризующие отражение Френеля [22,23] и соответственно равные

$$
R_{c}=\frac{\left(\frac{v_{\text {object }}}{v_{\text {medium }}}-1\right)^{2}}{\left(\frac{v_{\text {object }}}{v_{\text {medium }}}-1\right)^{2}}, \quad Q_{c}=\arcsin \left(\frac{v_{\text {medium }}}{v_{\text {object }}}\right),
$$

где $v_{\text {medium }}$ - относительный показатель преломления среды, окружающей исследуемый объект [24].

Численное решение уравнения (1) с граничным условием Робина (2) выполнено методом конечных разностей с использованием неявной разностной семиточечной схемы. В качестве критерия остановки итерационного процесса использовалось истечение заданного времени (в большинстве экспериментов моделировался временной интервал в $7 \mathrm{~ns}$ от момента инжектирования фотонов). Начальное приближение к функции $\phi(x, y, z, t)$ генерировалось с учетом положения источника фотонов на границе моделируемой биологической ткани и расчетного количества фотонов в падающем ультракоротком импульсе [23-25].

Для обеспечения возможности эффективного анализа влияния оптических свойств и геометрического расположения крупных поглощающих и рассеивающих неоднородностей в исследуемом объекте на формирование ВФРТ предлагается использовать нормированный максимум фотонной плотности (НМФП) (photon density normalized maximum, PDNM). Для его получения после завершения итерационного процесса функция нормируется относительно своего максимума $\phi_{\max }(x, y, z, t)$ :

$$
\phi_{\text {norm }}(x, y, z, t)=\frac{\phi(x, y, z, t)}{\phi_{\max }(x, y, z, t)},
$$

и подвергается следующему преобразованию [23]:

$$
\phi_{\mathrm{PDNM}}(x, y, z, t)=\left\{\begin{array}{l}
1, \quad \phi_{\text {norm }}(x, y, z, t) \geq P, \\
\phi_{\text {norm }}(x, y, z, t), \quad \text { иначе },
\end{array}\right.
$$

где $P$ - пороговый уровень при формировании НМФП, $0<P \leq 1$.

Предлагаемый подход имеет некоторые сходства (формирование и распространение в толще исследуемой ткани) с моделированием движения виртуального изотропного источника $[9,26,27]$, но принципиально отличается от него тем, что НМФП - не дополнение к функции источника фотонов, а результат постобработки уже вычисленного многомерного массива данных $\phi(x, y, z, t)$.

\section{Моделирование миграции фотонов в сильно рассеивающих средах на основе метода статистических испытаний Монте-Карло}

Существенно более точным, чем использование диффузионного приближения к УПИ, но и более требовательным к вычислительным ресурсам методом моделирования фотонного транспорта является метод статистических испытаний Монте-Карло. В многочисленных конкретных реализациях этого метода [7,10,25] вычисляются траектории случайного движения большого количества фотонов, причем оптический путь каждого из них отслеживается от момента инжектирования излучения в исследуемой объект до момента попадания соответствующего фотона на детектор, либо его „гибели“ вследствие потери большей части „веса“. При таком подходе к моделированию миграции фотонов в сильно рассеивающих средах оптический путь каждого фотона представляет собой совокупность актов взаимодействия (поглощение и рассеяние) на расстояниях свободного 
пробега. Расстояние между двумя такими актами вычисляется следующим образом [6,7]:

$$
L=-l_{\mathrm{ph}} \ln (1-\xi),
$$

где $L$ - длина свободного пробега фотона; $\xi$ псевдослучайное число из интервала от 0 до $1 ; l_{\mathrm{ph}}$ транспортная длина свободного пробега фотона:

$$
l_{\mathrm{ph}}=\frac{1}{\mu_{a}(x, y, z)+\mu_{s}^{\prime}(x, y, z)} .
$$

Вероятность, $p_{s}$, того, что для заданного фотона в заданной точке пространства актом взаимодействия будет акт рассеяния, вычисляется [25] как

$$
p_{s}=\frac{\mu_{s}(x, y, z)}{\mu_{a}(x, y, z)+\mu_{s}(x, y, z)} .
$$

Вероятность акта поглощения, $p_{z}$, вычисляется на основе уже известной $p_{s}$ следующим образом:

$$
p_{a}=1-p_{s} .
$$

В случае акта поглощения „вес“ фотона, $W$, уменьшается:

$$
W \rightarrow W-W \frac{\mu_{a}(x, y, z)}{\mu_{a}(x, y, z)+\mu^{\prime}(x, y, z)} .
$$

В случае акта рассеяния новое направление движения для фотона вычисляется следующим образом:

$$
\left\{\begin{array}{l}
x=x_{0}+\mu_{s} L \\
y=y_{0}+\mu_{y} L \\
z=z_{0}+\mu_{z} L
\end{array}\right.
$$

где $\mu_{x}, \mu_{y}, \mu_{z}-$ направляющие косинусы. В начальный момент времени направляющие косинусы равны

$$
\left\{\begin{array}{l}
\mu_{x}=0 \\
\mu_{y}=0 \\
\mu_{z}=0
\end{array}\right.
$$

затем при каждом акте рассеяния их величины пересчитываются [7,25] следующим образом:

$$
\left\{\begin{array}{l}
\mu_{x}^{\prime}=\frac{\sin \Theta}{\sqrt{1-\mu_{z}^{2}}}\left(\mu_{x} \mu_{z} \cos \varphi-\mu_{y} \sin \varphi\right)+\mu_{x} \cos \Theta \\
\mu_{y}^{\prime}=\frac{\sin \Theta}{\sqrt{1-\mu_{z}^{2}}}\left(\mu_{y} \mu_{z} \cos \varphi+\mu_{x} \sin \varphi\right)+\mu_{y} \cos \Theta \\
\mu_{z}^{\prime}=-\sin \Theta \cos \varphi \sqrt{1-\mu_{z}^{2}}+\mu_{z} \cos \Theta
\end{array}\right.
$$

где $\Theta$ - азимутально независимый угол рассеяния на основе фазовой функции Хени-Гринштейна, $\varphi$ полярный угол:

$$
\cos \Theta= \begin{cases}\frac{1}{2 g}\left(1+g^{2}-\left[\frac{1-g^{2}}{1-g+2 g \xi_{2}}\right]^{2}\right), & \text { если } g>0, \\ 2 \xi_{2}-1, & \text { если } g=0,\end{cases}
$$

$$
\varphi=2 \pi \xi_{3},
$$

где $\xi_{2}$ и $\xi_{3}-$ псевдослучайные числа из интервала от 0 до 1 .

В частном случае, когда угол падения фотона близок к нормали, направляющие косинусы $\mu_{x}, \mu_{y}, \mu_{z}$ вычисляются как

$$
\left\{\begin{array}{l}
\mu_{x}^{\prime}=\sin \Theta \cos \varphi \\
\mu_{y}^{\prime}=L \sin \Theta \sin \varphi \\
\mu_{z}^{\prime}=\operatorname{sign}\left(\mu_{z}\right) \cos \Theta
\end{array}\right.
$$

Для описания поведения фотона на границе моделируемой сильно рассеивающей среды используется вероятностное условие, $R\left(\Theta_{b}\right)$, внутреннего отражения $[8,9]$ :

$$
R\left(\Theta_{b}\right)=\frac{1}{2}\left[\frac{\sin ^{2}\left(\Theta_{b}-\Theta_{t}\right)}{\sin ^{2}\left(\Theta_{b}+\Theta_{t}\right)}+\frac{\tan ^{2}\left(\Theta_{b}-\Theta_{t}\right)}{\tan ^{2}\left(\Theta_{b}+\Theta_{t}\right)}\right] .
$$

где $\Theta_{b}-$ угол падения фотона на границу между исследуемой биологической тканью и окружающей средой, $\Theta_{t}-$ угол пропускания для падающих фотонов, равный

$$
\Theta_{t}=\arcsin \left(\frac{1}{v_{\text {medium }}}\right),
$$

После завершения вычисления независимых траекторий для всех рассматриваемых фотонов на их основе формируется функция $\phi(x, y, z, t)$. Для этого производится селекция актов взаимодействия по множеству точек пространства, $x, y, z$, и множеству моментов времени, $t$, после инжектирования фотонов. Причем момент времени, соответствующий $N_{\text {int }}$ - акту взаимодействия находится как

$$
t=\frac{\sum_{i=1}^{N_{\text {int }}} L_{i} v_{\text {object }}}{c_{0}} .
$$

Нормированный максимум фотонной плотности для распределений фотонной плотности, $\phi(x, y, z, t)$, вычисленных на основе метода статистических испытаний Монте-Карло, рассчитывается по тем же формулам (3) и (4), что и в случае с диффузионным приближением к УПИ.

\section{Результаты и обсуждение}

Вышеописанные математические модели миграции фотонов в сильно рассеивающих средах были практически реализованы с использованием программного пакета LabVIEW фирмы National Instruments. Для более эффективной работы модели на основе метода статистических испытаний Монте-Карло были применены параллельные вычисления на графических процессорах (NI GPU Analysis Toolkit).

Величина порогового уровня, $P$, при формировании НМФП была подобрана эмпирически и составила 99.5\% от максимума плотности фотонов $(P=0.995)$ в заданный момент времени. Более высокие значения $P$ делают 

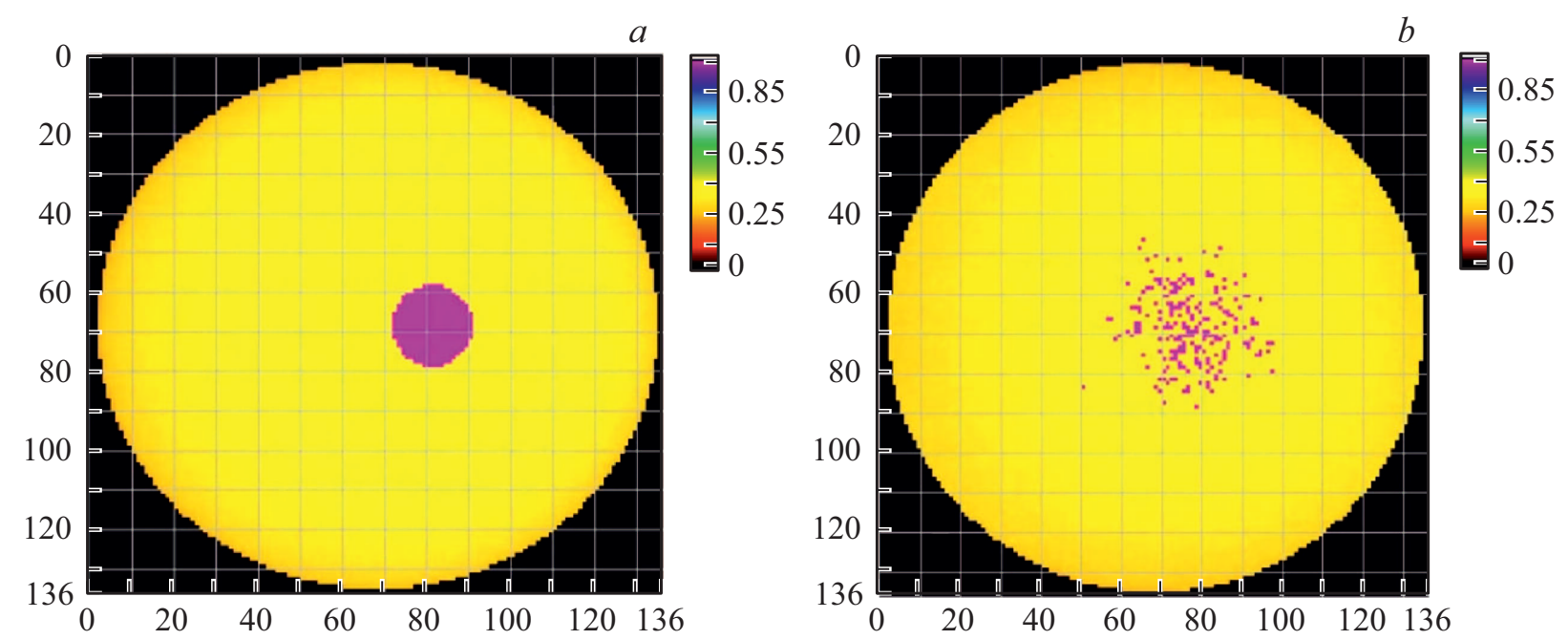

Рис. 1. Пространственные распределения фотонной плотности в срезах однородного цилиндрического объекта, вычисленные посредством $(a)$ численного моделирования на основе диффузного приближения к УПИ, $(b)$ численного моделирования методом статистических испытаний Монте-Карло. Срезы сделаны на полувысоте, фиолетовая область - нормированный максимум фотонной плотности $(P=0.995)$, цилиндрический объект характеризуется следующими оптическими свойствами: $\mu_{a}(x, y, z)=0.005 \mathrm{~mm}^{-1}, \mu_{s}^{\prime}(x, y, z)=0.5 \mathrm{~mm}^{-1}$.

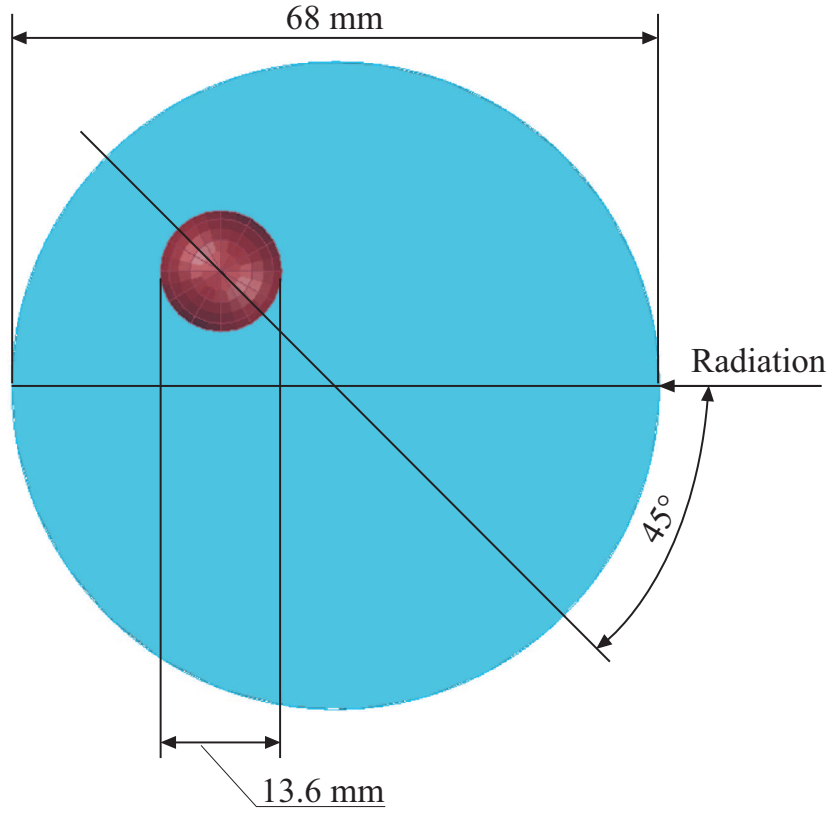

Рис. 2. Срез исследуемого цилиндрического фантома со сферической неоднородностью. Центр неоднородности расположен в плоскости полувысоты фантома.

НМФП излишне мелким и трудно различимым, более низкие значения этой величины также не удобны по причине сложностей анализа траектории движения [13].

Серия компьютерных экспериментов по моделированию миграции фотонов в однородных цилиндрических фантомах с различными оптическими свойствами и геометрическими характеристиками показала, что независимо от значений параметров поглощения, рассеяния, преломления и анизотропии, длины оси цилиндра и диаметра его основания НМФП движется к геометрическому центру исследуемого объекта $[10,13]$. На рис. 1 показаны распределения фотонной плотности с НМФП на полувысоте однородного цилиндрического фантома высотой и диаметром, равными $68 \mathrm{~mm}$ [21], вычисленные на основе диффузионного приближения к УПИ $(a)$ и метода статистических испытаний Монте-Карло $(b)$. Момент времени после инжектирования фотонов, $t=3 \mathrm{~s}$. Шаг сетки составляет $2 \mathrm{~mm}$. Оптические свойства фантома взяты из серии физических экспериментов [21,28] и приведены в подрисуночной подписи. Поведение НФМП по рис. 1 соответствует экспериментальным результатам, в которых все ВФРТ в однородном случае сходятся в прямую линию в области поздно пришедших фотонов (ППФ) [9,21,23]. С увеличением размеров исследуемого цилиндра увеличивается временной интервал, необходимый для достижения НМПФ центра объекта. Изменения в оптических свойствах также в основном влияют на продолжительность движения НМФП, но не на характер этого движения и тем более не на его конечную точку. Нормированный максимум фотонной плотности представляет собой замкнутую сферическую область при проведении вычислений на основе диффузионного приближения к УПИ и состоит из множества отдельных пиков при использовании метода Монте-Карло. Отличия связаны с особенностями проведения вычислений. Численное решение уравнения баланса фотонов в рассеивающей среде делает фотонный поток плавно распространяющимся во все стороны (такую модель еще называют моделью „капли“ по аналогии с диффузией чернил в воде). Вероятностные расчеты по методу Монте-Карло привели к формированию подобных распределений, но 
Таблица 1. Особенности движения НМФП в цилиндрическом объекте со сферической поглощающей неоднородностью: первая колонка - кратность превышения значением коэффициента поглощения неоднородности аналогичной характеристики для однородной части моделируемого объекта; вторая колонка $-V_{\text {avr }}^{a}$, средняя скорость движения НМФП с поправкой на изменение времени затухания оптического излучения; третья колонка $-L_{\text {res }}^{a}$, расстояние от конечной позиции НМФП до точки, симметричной центру поглощающей неоднородности относительно центра моделируемого объекта

\begin{tabular}{c|c|c}
\hline$\frac{\mu_{a \text { inh }}(x, y, z)}{\mu_{a \_h}(x, y, z)}$ & $\approx V_{\mathrm{avr}}^{a}, \mathrm{~mm} / \mathrm{ns}$ & $\approx L_{\mathrm{res}}^{a}, \mathrm{~mm}$ \\
\hline 2 & 5.9 & 6.1 \\
3 & 6.0 & 4.8 \\
4 & 6.1 & 3.5 \\
5 & 6.2 & 2.7 \\
6 & 6.3 & 1.9 \\
7 & 6.4 & 1.5 \\
8 & 6.4 & 1.3 \\
9 & 6.4 & 1.1 \\
10 & 6.5 & 0.9
\end{tabular}

Таблица 2. Особенности движения НМФП в цилиндрическом объекте со сферической рассеивающей неоднородностью: первая колонка - кратность превышения величиной коэффициента рассеяния неоднородности аналогичной характеристики для однородной части моделируемого объекта; вторая колонка $V_{\mathrm{avr}}^{s}$, средняя скорость движения НМФП с поправкой на изменение времени затухания оптического излучения; третья колонка $-L_{\text {res }}^{s}$, расстояние от конечной позиции НМФП до центра рассеивающей неоднородности

\begin{tabular}{c|c|c}
\hline$\frac{\mu_{s \operatorname{inh}}(x, y, z)}{\mu_{s, h}(x, y, z)}$ & $\approx V_{\mathrm{avr}}^{s}, \mathrm{~mm} / \mathrm{ns}$ & $\approx L_{\mathrm{res}}^{s}, \mathrm{~mm}$ \\
\hline 2 & 6.3 & 8.7 \\
3 & 6.8 & 7.1 \\
4 & 7.2 & 5.5 \\
5 & 7.7 & 3.6 \\
6 & 8.1 & 2.0 \\
7 & 8.9 & 1.2 \\
8 & 9.4 & 0.5 \\
9 & 9.9 & 0.3 \\
10 & 10.4 & 0.2
\end{tabular}

с небольшими всплесками. Плавное сглаживание распределений фотонной плотности, полученных при моделировании методом статистических испытаний МонтеКарло, повышает сходимость результатов двух моделей.

В случае наличия поглощающей неоднородности в исследуемом фантоме траектория миграции фотонов претерпевает существенные изменения. Независимо от пространственного расположения поглощающей неоднородности и ее коэффициента поглощения НМФП движется в направлении точки, которая симметрична центру поглощающей неоднородности относительно центра исследуемого объекта. На рис. 2 схематично пока показано внутреннее строение исследуемого неоднород- ного фантома. Неоднородность является сферической, имеет диаметр $13.6 \mathrm{~mm}$ (1/5 от диаметра основания фантома) и расположена под углом $\alpha=135^{\circ}$ по часовой стрелке относительно падающего излучения. Центры цилиндрического фантома и сферической неоднородности расположены в одной плоскости на расстоянии $17 \mathrm{~mm}$ (1/4 от диаметра основания фантома) друг от друга. На рис. 3 показаны результаты моделирования распределения фотонной плотности с НМФП в срезе исследуемого объекта, сделанном на полувысоте. По аналогии с однородным случаем рис. 3, $a$ показывает результаты вычислений на основе диффузионного приближения к УПИ, а рис. $3, b$ демонстрирует те же результаты, но на основе метода Монте-Карло. Момент времени после инжектирования фотонов, $t=2.5 \mathrm{~s}$. Шаг сетки составляет $2 \mathrm{~mm}$. Оптические свойства однородной части фантома и поглощающей неоднородности также взяты из серии физических экспериментов $[21,28]$ и приведены в подписи к рис. 3. Поведение НМФП по рис. 3 соответствует экспериментально полученным результатам [9,21], в которых при наличии поглощающей неоднородности в исследуемом объекте ВФРТ в области ППФ сходятся в прямые линии, причем нижняя из них будет соответствовать ближайшей к этой неоднородности точке детектирования. По аналогии с однородным случаем НМФП представляет собой замкнутую сферическую область (рис. 3,a) и множество отдельных пиков (рис. 3,b). Варьирование оптических свойств сферической неоднородности и однородной части цилиндрического объекта показало, что наибольшее влияние на скорость и выраженность движения НМФП оказывает отношение коэффициентов поглощения неоднородности и однородной части объекта. Чем в большее количество раз коэффициент поглощения неоднородности превышает аналогичный параметр для однородной части, тем быстрее движется НМФП и тем дальше он продвигается в направлении точки, симметричной центру неоднородности относительно центра исследуемого объекта (табл. 1).

Рассеивающая неоднородность в исследуемом фантоме меняет характер движения НМФП как относительно однородного случая (рис. 1), так и относительно случая с поглощающей неоднородностью (рис. 3). Нормированный максимум фотонной плотности движется в направлении центра рассеивающей неоднородности. На рис. 4 показан пример распределений фотонной плотности в срезе на полувысоте цилиндрического фантома. Рассеивающая неоднородность имеет те же размеры и местоположение (рис. 2), что и поглощающая в вышеописанном эксперименте. Момент времени после инжектирования фотонов, $t=4.5 \mathrm{~s}$. Шаг сетки составляет $2 \mathrm{~mm}$. Оптические свойства однородной части фантома и рассеивающей неоднородности $[21,28]$ приведены в подписи к рис. 4. Результаты моделирования на основе диффузионного приближения к УПИ и метода статистических испытаний Монте-Карло хорошо коррелируют с результатами физических эксперимен- 

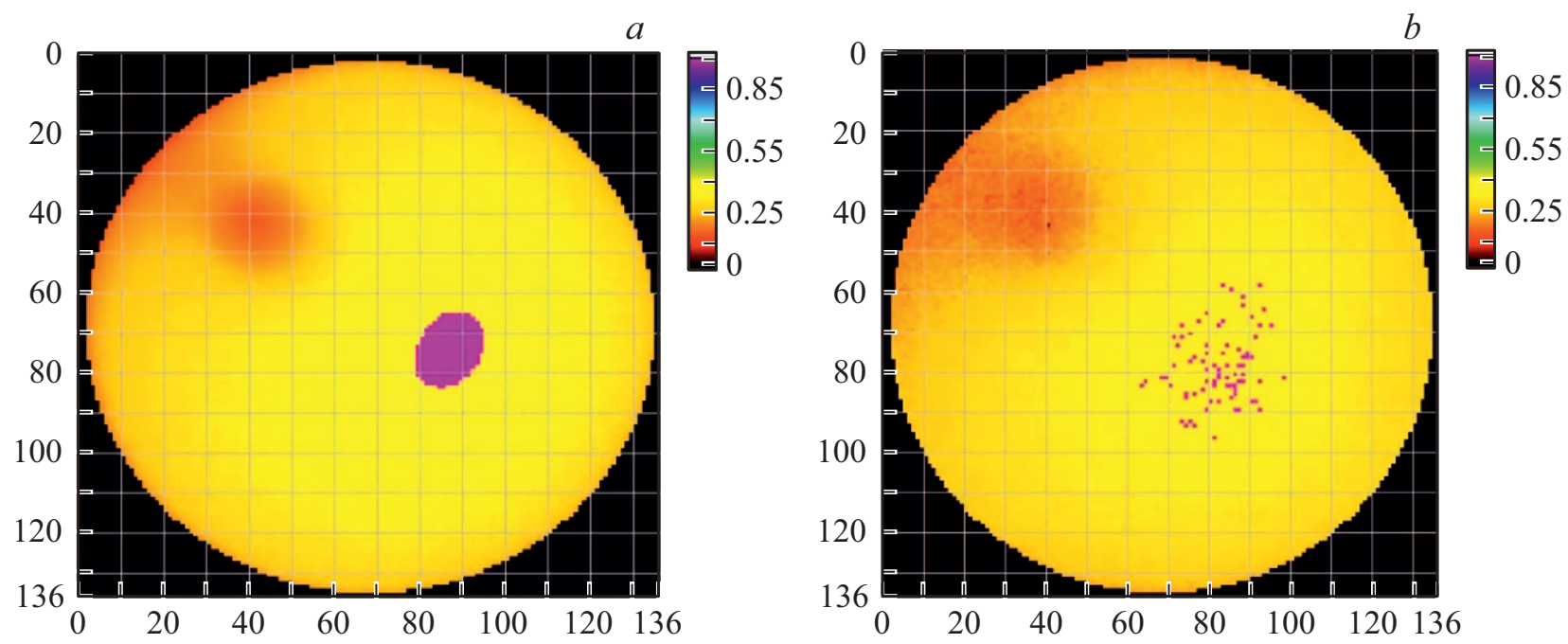

Рис. 3. Пространственные распределения фотонной плотности в срезах цилиндрического объекта со сферической поглощающей неоднородностью, вычисленные посредством $(a)$ численного моделирования на основе диффузного приближения к УПИ, (b) - численного моделирования методом статистических испытаний Монте-Карло. Срезы сделаны на полувысоте, фиолетовая область - нормированный максимум фотонной плотности $(P=0.995)$, цилиндрический объект однороден по значению коэффициента рассеяния $\mu_{s}^{\prime}(x, y, z)=0.5 \mathrm{~mm}^{-1}$, поглощающая неоднородность характеризуется значением коэффициента поглощения $\left(\mu_{a \_ \text {inh }}(x, y, z)=0.02 \mathrm{~mm}^{-1}\right)$, в 4 раза превышающим аналогичный показатель для остальных частей исследуемого объекта $\left(\mu_{a_{-} h}(x, y, z)=0.005 \mathrm{~mm}^{-1}\right)$.
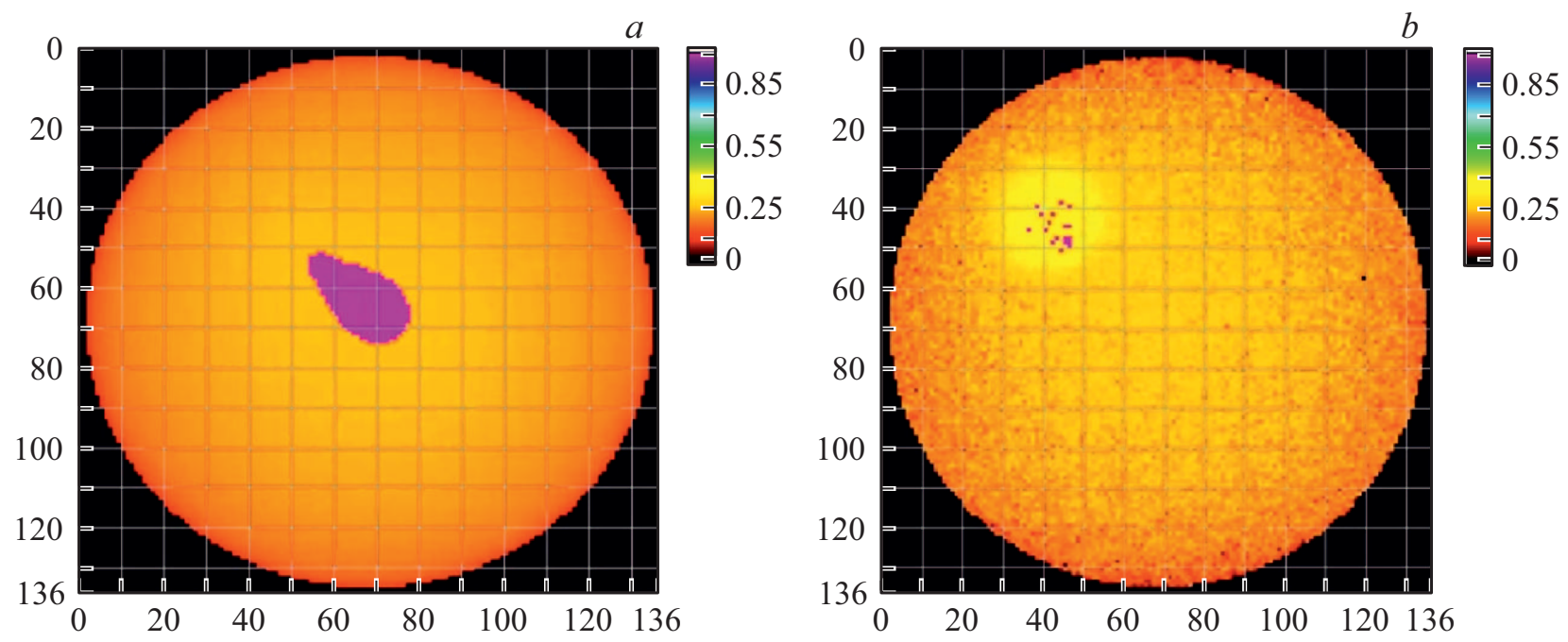

Рис. 4. Пространственные распределения фотонной плотности в срезах цилиндрического объекта со сферической рассеивающей неоднородностью, вычисленные посредством $(a)$ численного моделирования на основе диффузного приближения к УПИ, (b) численного моделирования методом статистических испытаний Монте-Карло. Срезы сделаны на полувысоте, фиолетовая область - нормированный максимум фотонной плотности $(P=0.995)$, цилиндрический объект однороден по значению коэффициента поглощения $\mu_{a}(x, y, z)=0.005 \mathrm{~mm}^{-1}$, рассеивающая неоднородность характеризуется значением коэффициента рассеяния $\left(\mu_{s}^{\prime} \operatorname{inh}(x, y, z)=2.0 \mathrm{~mm}^{-1}\right)$, в 4 раза превышающим аналогичный показатель для остальных частей исследуемого объекта

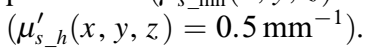

тов $[9,21]$, в которых рассеивающая неоднородность приводила к схождению ВФРТ в области ППФ в параллельные линии, причем верхняя из них соответствует ближайшей к этой неоднородности точке детектирования. Как и в случае с поглощающей неоднородностью, ключевое влияние на скорость и выраженность движения НМФП (табл. 2) оказывает отношение оптических свойств (в данном случае коэффициентов рассеяния) неоднородности и однородной части объекта. Небольшое запаздывание в движении НМФП по результатам моделирования на основе диффузионного приближения к УПИ относительно НМФП по результатам моделирования методом Монте-Карло может быть устранено посредством увеличения количества рассматриваемых 
фотонов (что также увеличивает продолжительность вычислений).

Отдельно следует отметить, что скорость полного затухания интенсивности излучения в экспериментах с поглощающими неоднородностями [21] приближенно на $20 \%$ выше, чем в экспериментах с однородными цилиндрами. Отдельные численные эксперименты с цилиндрическими объектами с рассеивающими неоднородностями показывают повышение времени полного затухания более чем на $30 \%$ по сравнению с однородным случаем.

\section{Заключение}

Сравнение результатов численного моделирования миграции фотонов в фантомах мягких биологических тканей на основе диффузионного приближения к УПИ и метода статистических испытаний Монте-Карло показывает высокую степень корреляции расчетных пространственных распределений фотонной плотности.

Постобработка результатов моделирования двумя вышеописанными методами с наложением и последующей визуализацией движения НМФП демонстрируют следующее:

- движение максимума фотонной плотности в направлении геометрического центра однородного объекта и, следовательно, приближенно одинаковый уровень интенсивности ВФРТ в области ППФ;

- движение максимума фотонной плотности в направлении точки, которая симметрична центру поглощающей неоднородности относительно центра моделируемого неоднородного объекта и, следовательно, более низкий уровень интенсивности ВФРТ в области ППФ для ближайшей к поглощающей неоднородности точке детектирования;

- движение максимума фотонной плотности в направлении центра рассеивающей неоднородности и, следовательно, более высокий уровень интенсивности ВФРТ в области ППФ для ближайшей к рассеивающей неоднородности точке детектирования;

- более высокую скорость затухания оптического излучения в экспериментах с поглощающими неоднородностями относительно однородных случаев;

- более низкую скорость затухания оптического излучения в экспериментах с рассеивающими неоднородностями относительно однородных случаев. Выявленные особенности движения НМФП могут быть полезны при разработке более эффективных методов формирования начального приближения к обратной задаче диффузионной оптической томографии. Дальнейшая работа будет сосредоточена на исследовании влияния ламинарного и турбулентного кровотока на ВФРТ и НМФП, в частности на изучении оптических неоднородностей с повышенной и пониженной оксигенацией крови.

\section{Благодарности}

Исследование выполнено за счет гранта Российского научного фонда (проект № 16-15-10327).

\section{Конфликт интересов}

Авторы заявляют об отсутствии конфликта интересов.

\section{Список литературы}

[1] Hoshi Y., Yamada Y. // J. Biomed. Opt. 2016. V. 21(9). Art. No. 091312.

[2] Angelo J.P., Chen S-J., Ochoa M., Sunar U., Gioux S., Intes X. // J. Biomed. Opt. 2018. V. 24(7). Art. No. 071602.

[3] O'Sullivan T.D., Cerussi A.E., Tromberg B.J., Cuccia D.J. // J. Biomed. Opt. 2012. V. 17(7). Art. No. 071311.

[4] O'Leary M.A., Boas D.A., Chance B., Yodh A.G. // Opt. Lett. 1995. V. 20(5). P. 426.

[5] Dehghani H., Srinivasan S., Pogue B.W., Gibson A. // Phil. Trans. Roy. Soc. A. 2009. V. 367. P. 3073.

[6] Yamada Y., Okawa S. // Opt. Rev. 2014. V. 21(3). P. 185.

[7] Fang Q., Boas D.A. Monte-Carlo // Opt. Express. 2009. V. 17(22). P. 20178.

[8] Schweiger M., Arridge S. // J. Biomed. Opt. 2014. V. 19(4). Art. No. 040801.

[9] Proskurin S.G., Yamada Y., Takahashi Y. // Opt. Rev. 1995. V. 2(4). P. 292

[10] Потлов А.Ю., Фролов С.В., Проскурин С.Г. // Опт. и спектр. 2016. T. 120. № 1. C. 15; Potlov A.Yu., Frolov S.V., Proskurin S.G. // Opt. Spectr. 2016. V. 120 (1). P. 9.

[11] Cao N., Nehorai A. // Opt. Express. 2007. V. 15(3). P. 896.

[12] Коновалов А.Б., Власов В.В., Калинцев А.Г., Кравценюк О.В., Любимов В.В. // Квант. электрон. 2006. Т. 36(11). C. 1048. Konovalov A.B., Vlasov V.V., Kalintsev A.G., Kravtsenyuk O.V., Lyubimov V.V. // Quant. Electron. 2006. V. 36(11). P. 1048.

[13] Potlov A.Yu., Frolov S.V., Proskurin S.G. // Computational Biomechanics for Medicine / Ed. by Joldes G., Doyle B., Wittek A., Nielsen P.M.F., Miller K. Switzerland: Springer Int. Publ. 2016. P. 51.

[14] Гуслякова О.И., Ленгерт Е.В., Аткин В.С., Тучин В.В., Свенская Ю.И. // Опт. и спектр. 2019. Т. 126. № 5. C. 620. Gusliakova O.I., Lengert E.V., Atkin V.S., Tuchin V.V., Svenskaya Yu.I. // Opt. Spectr. 2019. V. 126(5). P. 539.

[15] Pogue B.W., Patterson M.S. // J. Biomed. Opt. 2006. V. 11(4). Art. No. 041102.

[16] Tavakoli B., Zhu Q. // J. Biomed. Opt. 2013. V. 18(1). Art. No. 16006.

[17] Ban H.Y., Schweiger M., Kavuri V.C., Cochran J.M., Xie L., Busch D.R., Katrašnik J., Pathak S., Chung S.H., Lee K., Choe R., Czerniecki B.J., Arridge S.R., Yodh A.G. // Medical Physics. 2016. V. 43(7). Art. No. 4383.

[18] Hoi J.W., Kim H.K., Fong C.J., Zweck L., Hielscher A.H. // Biomed. Opt. Express. 2018. V. 9(11). P. 5597.

[19] Giraev K.M., Ashurbekov N.A., Kobzev O.V. // Tech. Phys. Lett. 2003. V. 29(11). P. 901.

[20] Bi B., Han B., Han W., Tang J., Li L. // Comput. Mathematic. Meth. in Medicine. 2015. Art. No. 286161.

[21] Proskurin S.G., Potlov A.Yu., Frolov S.V. // Quant. Electron. 2015. V. 45(6). P. 540. 
[22] Konovalov A.B., Genina E.A., Bashkatov A.N. // J.Biomed. Photon. Engineer. 2016. V. 2(2). Art. No. 020202.

[23] Potlov A.Yu., Frolov S.V., Proskurin S.G. // J. Phys. Conf. Ser. 2018. V. 1084. Art. No. 012012.

[24] Gantri M. // Comput. Mathematic. Meth. in Medicine. 2014. Art. No. 814929.

[25] Berrocal E., Sedarsky D.L., Paciaroni M.E., Meglinski I.V., Linne M.A. // Opt. Express. 2009. V. 17(16). P. 13792.

[26] Wang L.-H., Jacques S.L. // Comput. Meth. Progr. in Biomed. 2000. V. 61(3). P. 163.

[27] Berrocal E., Meglinski I.V., Greenhalgh D.A., Linne M.A. // Las. Phys. Lett. 2006. V. 3(9). P. 464.

[28] Potlov A.Yu., Frolov S.V., Proskurin S.G. // Las. Phys. 2015. V. 25. Art. N 035601. 